\title{
Relaciones entre calidad de vida y factores sociodemográficos y de salud en adultos mayores colombianos
}

\author{
Relations between quality of life, sociodemographic \\ and health factors in Colombian elderly
}

\author{
Stefano Vinaccia Alpi , Fernando Riveros Munévar ${ }^{2}$, \\ Leonardo García-Rincón ${ }^{3}$, Japcy Margarita Quiceno ${ }^{4}$, \\ Olga Viviana Martínez ${ }^{l}$, Alejandra Martínez ${ }^{1}$ y Kimberly Reyes ${ }^{1}$
}

\begin{abstract}
RESUMEN
El objetivo de este trabajo fue analizar las relaciones entre calidad de vida y factores sociodemográficos y de salud en 150 adultos mayores colombianos. Fue un estudio descriptivo correlacional, con ejercicios de comparación de grupos a los cuales se aplicaron diversos instrumentos que miden las variables de interés. Los resultados mostraron puntuaciones altas en placer y resiliencia, y bajas en ansiedad, depresión y dolor. El 92.7\% de los participantes mostró riesgo de dependencia de otros para la ejecución de sus funciones. Las personas viudas y casadas tuvieron mayores puntuaciones en placer. Aquellos que vivían solos tuvieron una calidad de vida desfavorable; quienes tenían una actividad laboral y estudiaban reportaron mayor placer y autorrealización. Las personas pertenecientes al Sistema de Identificación de Potenciales Beneficiarios de Programas Sociales exhibieron menor resiliencia, calidad de vida, autorrealización y control, y una mayor percepción de dolor. Finalmente, hubo relaciones positivas entre calidad de vida y resiliencia. La ansiedad correlacionó negativamente con control y resiliencia, y la depresión con control, autorrealización, calidad de vida y resiliencia. Se concluye que los adultos mayores refieren mejor calidad de vida en condiciones tales como realizar alguna actividad laboral o académica, contar con una afiliación a un sistema de salud y vivir en compañía.
\end{abstract}

Palabras clave: Calidad de vida; Resiliencia; Ansiedad/depresión; Riesgo de dependencia; Actividades de la vida diaria; Dolor.

\begin{abstract}
The aim of this descriptive-correlational study, with a group comparison, was to evaluate the relationships between quality of life, sociodemographic and health factors in 150 Colombian elderly adults residents of Bogotá. Participants completed a series of questionnaires in order to measure the variables of interest. The results showed high scores on pleasure and resilience, and low scores on anxiety, depression, and pain. Almost 93\% of participants, however, showed a risk of dependency on others for functioning properly. Older widows and married people had higher scores on pleasure and people living alone had a dimished quality of life. Similarly, participants who worked and studied reported higher pleasure and self-realization. Participants belonging to social welfare (SISBEN) showed lower resilience, quality of life, self-realization, and control, as well as greater perception of pain. Finally, there were positive associations between quality of life and resilience; the anxiety response was negatively correlated with control and resilience, and depression negatively correlated with control, self-actualization, quality of life, and resilience. In conclusion, older adults
\end{abstract}

\footnotetext{
${ }^{1}$ Fundación Universitaria Sanitas, Carrera 7, Núm. 173-64, Bogotá, Colombia, tel. (57)15-89-53-77, correo electrónico: vinalpi47@hotmail.com. Artículo recibido el 19 de mayo y aceptado el 28 de agosto de 2017.

${ }^{2}$ Universidad de San Buenaventura, Carrera 8H, Núm. 172, raya 20, Bogotá, Colombia.

${ }^{3}$ Universidad El Bosque, Av. Carrera 9, Núm. 131 A-02, Edificio Fundadores, $2^{\circ}$ Piso, Bogotá, Colombia.

${ }^{4}$ Universidad de Medellín, Carrera 87, Núm. 30 - 65, Medellín, Colombia.
} 
report better quality of life when performing some work or academic activity, have healthcare, and live in company.

Key words: Quality of life; Resilience; Anxiety/depression; Risk of dependence; Activities of daily living; Pain.

\section{INTRODUCCIÓN}

$\mathrm{E}$ 1 envejecimiento poblacional es un fenómeno global que afecta a numerosos países desarrollados y en vías de desarrollo. En el año 2015 había 901 millones de adultos mayores de 60 años, un aumento de 48\% respecto al porcentaje correspondiente al año 2000, calculado en 607 millones. Se prevé que esta cifra crezca en $56 \%$ en el año 2030 y que en 2050 tenga el doble del tamaño que tenía en 2015, llegando a casi 2.1 mil millones (Organización Mundial de la Salud [OMS], 2015).

En Colombia, según Dulcey, Arrubla y Sanabria (2013), los 4,964,793 adultos mayores de 60 años correspondían en 2013 a $10.5 \%$ de la población; de ese total, 650,000 sobrepasaban los 80 años. El Global Aging Institute [CGA] (2017) señala que entre los años 2010 y 2036 los mayores de 65 años en Colombia pasarán de ser 6\% de la población a $15 \%$, un porcentaje que Estados Unidos demoró 69 años en alcanzar, y 115 Francia. De este porcentaje, se calcula que solo una de cada cuatro personas en Colombia recibirá pensión, y una de cada siete tratándose de mujeres; además, 44\% de los adultos mayores de 65 años serán de bajos recursos - la tasa más alta de toda América-; por otro lado, las proyecciones poblacionales plantean que en el año 2050 Colombia tendrá cerca de 14.1 millones de adultos mayores de 60 años viviendo en su territorio, equivalente a casi una cuarta parte de la población (Fundación Saldarriaga Concha, 2015).

La calidad de la atención que reciben las personas que envejecen en Colombia varía según el lugar donde viven, pues aquellas que residen en las zonas altamente urbanizadas pueden recibir una atención de buena calidad, mientras que los habitantes de las zonas rurales y en las regiones del sur y del norte están expuestos al desempleo, los bajos ingresos, la desigualdad de acceso a la aten- ción médica, el tráfico de drogas y los conflictos armados (Gómez, Curcio y Duque, 2009).

El reto para los estudios sobre el envejecimiento es entender cómo las condiciones asociadas con él no únicamente dependen de variables de tipo físico o fisiopatológicas que pueden producir enfermedades o fragilidad progresiva, sino también de factores psicológicos, cognitivos y sociales que, por el contrario, podrían contribuir a un proceso positivo en la vejez en el que la salud, el bienestar, las fortalezas personales y el placer pueden ser parte de la calidad de vida (Fontes y Neri, 2015).

Por otro lado, la calidad de vida, como un constructo multidimensional, es potencialmente influida positivamente por diversas variables, incluyendo las sociodemográficas (ingreso familiar y nivel de escolaridad) (Poli et al., 2016), la percepción del apoyo familiar (Hoogendijk et al., 2016), las relaciones con la familia y los amigos (Cornwell, 2016), la vida social y las actividades de ocio (Nimrod y Shrira, 2016), la resiliencia y las emociones positivas (Cárdenas y López, 2011; Smith y Hollinger-Smith, 2015; Zen y Shen, 2010), y negativamente por otras variables, como los síntomas depresivos, la ansiedad, el dolor, la fragilidad y el estrés (Gómez, Curcio y Henao, 2012).

Los estudios desarrollados en Colombia han investigado la calidad de vida de adultos mayores en diferentes contextos y estados físicos (Cano, Borda, Arciniegas y Parra, 2014; Cardona, Álvarez y Pastrana, 2012; Cardona, Estrada y Agudelo, 2006), su autopercepción (Ocampo, Zapata, Cubides, Curcio y Villegas, 2013), las relaciones entre el estatus socioeconómico y la calidad de vida (Lucumi, Gómez, Browson y Parra, 2014), la depresión (Bacca, Gonzales y Uribe, 2005), la fragilidad (Curcio, Henao y Gómez, 2014), la discapacidad (Agudelo, Ayala y Ríos, 2009) y la resiliencia (Bennett, Reyes, Altamar y Soulsby, 2016).

También está en proceso de desarrollo el proyecto de investigación SABE Colombia, con el cual se pretende medir la antropometría, la presión arterial, la función física y las medidas bioquímicas y hematológicas, las características de los cuidadores familiares y la calidad de vida, entre otras variables, de más de 23 mil adultos mayores (Gómez, Corchuelo, Curcio, Calzada y Méndez, 2016). 
En 2002, la OMS introdujo el concepto de "envejecimiento activo", definiéndolo como el proceso en el cual se optimizan las oportunidades para promover la salud, la participación y la seguridad de las personas que están en dicho proceso de envejecimiento. Al respecto, Kahana, Kelley-Moore y Kahana (2012) desarrollaron el Modelo Proactivo de Éxito en el Envejecimiento, con base en el cual la calidad de vida de estas personas podría relacionarse con la utilización de recursos internos y externos para afrontar de una forma proactiva los retos que conlleva el envejecimiento (cf. Fedeli, 2014).

A partir de lo anterior, y ante la escasa investigación nacional en torno a los datos de calidad de vida en adultos mayores y sus variables relacionadas, el objetivo de este estudio descriptivo, correlacional y con ejercicios de comparación de grupos (Hernández, Fernández y Baptista, 2010), fue analizar las relaciones entre la calidad de vida y factores sociodemográficos y de salud en adultos mayores colombianos.

\section{MÉTODO}

\section{Participantes}

Por medio de un muestreo no aleatorio por sujetos disponibles y teniendo en cuenta como criterios de inclusión que fueran mayores de 60 años, residentes en la ciudad de Bogotá, sin alteraciones neurocognitivas, participaron en este estudio 150 adultos mayores de entre 60 y 98 años de edad $(\mathrm{M}=72.4$, D.T. $=7.3)$, de los cuales 68 fueron hombres $(45.3 \%)$ y 82 mujeres $(54.7 \%)$, La mayoría pertenecían al estrato socioeconómico colombiano de 1 a 4 (bajo y medio). Gran parte de la muestra era casada $(48 \%), 20 \%$ era viuda y $13.3 \%$ soltera. De los participantes, $78.7 \%$ vivían con familiares, mientras que $19.3 \%$ vivían solos. Cabe señalar que, en Colombia, las personas pueden estar adscritas a diferentes sistemas de salud, como el Sistema de Identificación y Clasificación de Potenciales Beneficiarios de Programas Sociales (SISBEN), las Entidades Promotoras de Salud (EPS) y la medicina prepagada (servicio de salud que garantiza y cubre más de lo que ofrece el Plan Obligatorio de Salud). En este estudio, 78.7\% de los participantes estaba adscrito a una EPS, $17.3 \%$ al SISBEN, $4 \%$ a medicina prepagada y $60 \%$ consumía medicamentos regularmente. Cabe señalar que la muestra se obtuvo mediante un convenio con el Instituto Distrital de Recreación y Deporte (IDRD) de la ciudad de Bogotá.

\section{Instrumentos}

Escala de Calidad de vida CASP-19. Desarrollada por Hyde, Wiggins, Higgs y Blane (2003), en esta investigación se usó la versión experimental colombiana de Vinaccia (2010), que comprende 19 ítems que se agrupan en cuatro dimensiones: control, autonomía, placer y autorrealización, y además una escala total. El sistema de respuesta es de tipo Likert de cuatro puntos, que va de "frecuentemente" a "nunca". En este estudio, la escala obtuvo un coeficiente alfa de Cronbach de 0.815.

Cuestionario de Resiliencia del CLHLS. La versión experimental colombiana de Vinaccia (2010), derivada del Chinese Longitudinal Healthy Longevity Survey (Zeng y Shen, 2010), es un cuestionario tipo Likert de cinco ítems que mostró un coeficiente alfa de Cronbach de 0,755.

Cuestionario Breve de Tamizaje de Depresión y Ansiedad (PHQ-4). Desarrollado por Kroenke, Spitzer, Williams y Lowe (2009), retoma del DSMIV los dos criterios principales para estas patologías y los puntúa en una escala de 0 ("casi ningún día") a 3 ("casi todos los días"), con una puntuación máxima de 12. En el presente estudio se usó la versión estandarizada en Colombia de Kocalevent, Finck, Jiménez, Sautier y Hinz (2014), con un índice de confiabilidad de 0.84 . El coeficiente alfa de Cronbach en el presente estudio fue de 0.636.

Cuestionario de Barber (CB). Desarrollado por Barber, Wallis y McKeating (1980), se empleó aquí la versión española de Larragoita y Larragoita (1996). El CB es una escala autoadministrada que mide el riesgo de dependencia a otras personas. Comprende nueve ítems que se responden en un sistema de verdadero/falso, con puntuaciones de 0 a 1 , donde un punto o más sugiere situaciones de riesgo. En esta investigación, el cuestionario mostró un coeficiente alfa de Cronbach de .668.

Índice de Katz. Elaborado por Katz, Ford, Moscowitz, Jackson y Jaffe (1963), se utilizó en este estudio la versión española de Álvarez et al. 
(1992). Este instrumento mide la valoración que hace la persona de las actividades de la vida diaria, y está heteroadministrado con seis ítems dicotómicos y con puntuaciones que van de 0 (cuando la actividad es realizada de forma independiente) a 1 (cuando la actividad se realiza con ayuda o no se realiza). La puntuación se estima por grados, siendo los grados A-B o 0-1 puntos iguales a la ausencia de incapacidad o incapacidad leve: los grados C-D o 2-3 puntos a una incapacidad moderada, y los grados E-G o 4-6 puntos a una incapacidad severa. En este estudio, los datos de confiabilidad según el coeficiente alfa de Cronbach arrojaron un coeficiente alfa de .914 .

Escala Visual Analógica (EVA). Este instrumento se compone del dibujo de una línea vertical de diez centímetros en la que la persona indica el nivel de percepción de dolor sobre la misma, de manera que va de 1 a 10 puntos. Las puntuaciones cercanas al extremo inferior de la línea indican una percepción menor de síntomas de dolor y las que se hallan cerca del extremo superior de la línea muestran una mayor percepción.

Ficha de datos sociodemográficos. La ficha de datos sociodemográficos permite recoger información sobre edad, género, estrato, ocupación, personas con quién vive el participante y condiciones de salud.

\section{Procedimiento}

Antes de empezar la fase de campo de la investigación, que implicaba la aplicación de las pruebas psicométricas, se llevó a cabo el proceso de adaptación cultural de las mismas, según los criterios de Alexandre y Guirardello (2002). Con el CAPS- 19, el CLHS, el Barber y el Índice de Katz el trabajo de adaptación cultural consistió en la aplicación de las pruebas a un grupo de diez adultos mayores de ambos sexos que, luego de responderlas, fueron entrevistados para identificar palabras o preguntas de difícil comprensión, evaluar su aceptabilidad y hacer comentarios sobre ellas de una forma general, registrándose además el tiempo necesario para completarlas y la comprensión del sistema de respuesta de cada ítem. No se ha- 1laron dificultades que ameritaran hacer cambios, pues los diez participantes reportaron entender los ítems en todos los instrumentos psicométricos así evaluados. Luego de la explicación del propósito de la investigación, de la aceptación y firma del formato de consentimiento informado por parte de cada adulto mayor, se aclararon también algunas consideraciones éticas, como la confidencialidad, y se diligenciaron las pruebas de manera individual con la ayuda del investigador a cargo. La duración aproximada de la aplicación de los instrumentos psicométricos fue de una hora y treinta minutos. Los datos obtenidos se integraron a una base de datos y se analizaron a través del paquete estadístico SPSS, versión 24.

\section{RESULTADOS}

Para el análisis descriptivo de la edad y las variables de salud (calidad de vida, resiliencia, ansiedad y depresión, riesgo de dependencia, actividades de la vida diaria y dolor) se utilizó la media como medida de tendencia central. Asimismo, se calcularon la desviación típica y las puntuaciones mínimas y máximas de las diferentes dimensiones de los instrumentos psicométricos utilizados; respecto a las variables nominales, se emplearon porcentajes (riesgo de dependencia e Índice de Katz para actividades de la vida diaria).

Para determinar la distribución de las variables numéricas se usó el análisis de Kolmogorov-Smirnov, encontrándose que todas ellas se distribuyeron de forma no normal $(p<.05)$, por lo cual los análisis estadísticos realizados para cumplir con los objetivos de la investigación fueron hechos a partir de pruebas no paramétricas. Para el análisis de las diferencias de las variables de salud según los actores sociodemográficos se utilizaron la prueba de U de Mann-Whitney (umw) y la de Kruskal-Wallis (kw) en el caso de las variables polinómicas; por último, se empleó la prueba de Sperman para el análisis de correlación entre las variables de salud y las numéricas del estudio. En la Tabla 1 se muestran los datos descriptivos de las variables numéricas en salud. 
Tabla 1. Estadísticos descriptivos de las variables CVRS, resiliencia, ansiedad, depresión y dolor.

\begin{tabular}{|l|r|r|r|r|}
\hline Variables & Media & D.T. & Mín. & Máx. \\
\hline CASP-19: CVRS & & & & \\
\hline Control & 7.87 & 2.93 & 0 & 12 \\
\hline Autonomía & 8.96 & 2.74 & 0 & 15 \\
\hline Placer & 10.04 & 2.36 & 0 & 12 \\
\hline Autorrealización & 13.09 & 3.63 & 0 & 18 \\
\hline Escala total & 39.96 & 9.06 & 0 & 55 \\
\hline CLHLS: Resiliencia & 19.10 & 4.28 & 0 & 25 \\
\hline PHQ-4: Ansiedad & 1.13 & 1.29 & 0 & 6 \\
\hline PHQ-4: Depresión & 1.09 & 1.28 & 0 & 6 \\
\hline VAS: Dolor & 2.19 & 2.20 & 0 & 10 \\
\hline
\end{tabular}

Al compararse la media con los mínimos y máximos, los datos de la tabla muestran puntajes medios en control, autonomía y autorrealización, y puntajes altos en placer y resiliencia, así como bajos en ansiedad, depresión y dolor en la escala general de calidad de vida. En la Tabla 2 se muestra la tendencia en las variables Riesgo de dependencia y Actividades de la vida diaria.

Tabla 2. Frecuencias en el riesgo de dependencia y las actividades de la vida diaria.

\begin{tabular}{|l|r|r|c|}
\hline \multicolumn{1}{|c|}{ Riesgo dependencia } & Frec. & \multicolumn{1}{c|}{ \% } & \% acum. \\
\hline Actividades de la vida diaria & 11 & 7.3 & 7.3 \\
\hline Riesgo de dependencia & 139 & 92.7 & 100.0 \\
\hline Total & 150 & 100.0 & \\
\hline Total AVD & 128 & 85.3 & 85.3 \\
\hline 0 & 10 & 6.7 & 92.0 \\
\hline 1 & 1 & .7 & 92.7 \\
\hline 2 & 1 & .7 & 93.4 \\
\hline 3 & 3 & 2.0 & 95.4 \\
\hline 4 & 4 & 2.7 & 98.1 \\
\hline 5 & 3 & 1.9 & 100.0 \\
\hline 6 & 150 & 100.0 & \\
\hline Total & & &
\end{tabular}

Los datos en la Tabla 2 refieren que $92,7 \%$ de la muestra presentó un riesgo de dependencia a otros para la ejecución de sus funciones, y en el Katz se halló que $92 \%$ refirió no tener incapacidad o padecía una incapacidad leve, 1,4\% mostró una incapacidad moderada y el $6,7 \%$ restante, una discapacidad severa para llevar a cabo sus actividades cotidianas.

\section{Diferencias de las variables de salud según las características sociodemográficas}

Las variables sociodemográficas que presentaron diferencias significativas respecto a las variables de salud fueron, a saber: género, estado civil, personas con quienes vive, ocupación, tratamiento farmacológico y sistema de salud adscrito.

Género. La variable de género se analizó a través de la prueba umw. No se encontraron diferencias significativas $(p>.05)$ con las puntuaciones de las variables de salud (tiempo de diagnóstico; riesgo de dependencia; CAPS-19 control, autonomía, placer, autorrealización, placer y escala total; actividades de la vida diaria; resiliencia, y dolor). Sin embargo, se hallaron diferencias en los promedios de la variable de ansiedad (hombres $=68.48$ y mujeres $=81.32 ; p<.05)$, lo que sugiere que los hombres que participaron en el estudio mostraron ser más ansiosos que las mujeres.

Estado civil. La variable "estado civil”, de naturaleza nominal politómica de cinco categorías (soltero, casado, separado, unión libre y viudo), se analizó con la prueba kw. Se encontró que la dimensión de placer del CASP-19 exhibió diferencias significativas $(\mathrm{kw}=10.35 ; p<.05)$ con las categorías de viudo $(\mathrm{M}=87.07)$, casado $(\mathrm{M}=78.47)$ y soltero $(M=51.05)$, indicando que las mujeres viudas y casadas tuvieron mayores puntuaciones respecto a las personas solteras.

Con quién vive. Esta variable contó con tres categorías (solo, con familiares, con amigos), y el análisis se realizó a partir del estadístico no paramétrico kw. Se encontraron diferencias significativas de la dimensión de control del CASP-19 $(\mathrm{kw}=7.74$ : $p<.01)$ con las categorías con amigos $(\mathrm{M}=81) \mathrm{y}$ con familiares $($ Media $=79.15)$; la media más baja la obtuvieron quienes vivían solos $(\mathrm{M}=58.17)$. Asimismo, se encontraron diferencias significativas de la dimensión de autorrealización del CASP-19 $(\mathrm{kw}=8.78: p<.05)$, con las categorías con amigos $(\mathrm{M}=95.50)$, seguida de las personas que viven con la familia $(\mathrm{M}=79.51)$; las puntuaciones medias más bajas las tuvieron quienes vivían solos $(\mathrm{M}=54.28)$. Finalmente, la escala total del CASP-19 tuvo diferencias significativas $(\mathrm{kw}=7.41 p<.05)$, con las categorías vivir con amigos $(\mathrm{M}=124) \mathrm{y}$ con familiares $(\mathrm{M}=79.51)$, obteniendo las pun- 
tuaciones medias más bajas quienes vivían solos $(\mathrm{M}=51.22)$.

Ocupación. Esta variable constó de seis categorías (empleado, trabajador independiente, jubilado, desempleado, ama de casa y trabaja y estudia). El análisis se realizó a partir del estadístico no paramétrico kw. Hubo diferencias significativas de la dimensión placer del CASP-19 $(\mathrm{kw}=16.75: p<.01)$ con las categorías trabaja y estudia $(\mathrm{M}=121.50)$, empleado $(\mathrm{M}=80.58)$, trabajador independiente $(\mathrm{M}=56.15)$, jubilado $(\mathrm{M}=79.80)$, desempleado $(\mathrm{M}=54)$ y ama de casa $(\mathrm{M}=87.91)$. Hubo también diferencias significativas de la dimensión de autorrealización del CASP-19 $(\mathrm{kw}=12.55: p<.05)$ con la categoría empleado, que tuvo la puntuación media más alta $(M=97.50)$ respecto a quienes trabajaban y estudiaban $(\mathrm{M}=95.5)$ y los desempleados $(M=54.79)$. Por último, la escala total del CASP-19 también tuvo diferencias significativas $(\mathrm{kw}=12.48: p<.05)$ con las categorías trabaja y estudia $(\mathrm{M}=124)$, seguidas de empleado $(\mathrm{M}=$ 94.42) y desempleado $(\mathrm{M}=52.47)$.

Tratamiento farmacológico. Esta variable sociodemográfica, de naturaleza nominal dicotómica, se analizó con la prueba umw. Se encontraron diferencias significativas a partir del diagnóstico (umw $=508: p<.05)$, habiendo un mayor puntaje promedio de diagnóstico en los que se hallaban en tratamiento farmacológico (M Con Tratamiento $=98.16:$ M Sin Tratamiento $=38.97)$. En las demás variables de estudio no se encontraron diferencias significativas.

Sistema de salud adscrito. Esta última variable sociodemográfica se refiere al sistema de salud en el que se encontraban afiliados los participantes del estudio.

Al respecto, se encontraron diferencias significativas con la resiliencia $(\mathrm{kw}=11.20: p<.01)$, puntuando más alto en aquellos que se encontraban afiliados a la EPS $(M=81.18)$, seguidos de los que estaban en medicina prepagada $(\mathrm{M}=75.25)$; los puntajes más bajos fueron los de quienes se encontraban afiliados a SISBEN $(M=49.77)$. También se encontraron diferencias significativas con la variable escala total del CASP-19 $(\mathrm{kw}=9.52$ : $p<.01)$; en esta, los puntajes más altos fueron de aquellos que estaban vinculados a la medicina prepagada $(\mathrm{M}=105.67)$, seguidos de los que estaban suscritos a la EPS $(\mathrm{M}=78.57)$; el punta- je más bajo de calidad de vida lo obtuvieron las personas del SISBEN $(\mathrm{M}=54.60)$. La dimensión de autorrealización del CASP-19 fue otra variable que estuvo asociada de forma significativa el régimen de salud $(\mathrm{kw}=9.86: p<.01)$, hallándose que las personas que estaban vinculadas a la medicina prepagada tenían mayores niveles de autorrealización $(\mathrm{M}=112.17)$, seguidos de los que estaban vinculados a la EPS $(\mathrm{M}=77.89)$; los niveles más bajos fueron los de aquellos que pertenecían al SISBEN $(\mathrm{M}=56,19)$. También se encontró una asociación significativa con la dimensión de control del CASP-19 (kw $=6.54: p<.05)$, en la cual las personas que contaban con medicina prepagada tenían mayores puntajes $(\mathrm{M}=88)$, seguidos de los que estaban vinculados a EPS $(\mathrm{M}=79.03)$; nuevamente, los puntajes más bajos fueron los de quienes estaban en el régimen contributivo $(\mathrm{M}=$ 56.19). El dolor fue otra variable con la que se encontró asociación significativa $(\mathrm{kw}=5.98: p<.05)$, lo que indica que las personas que pertenecían al régimen contributivo tenían mayores niveles de dolor $(\mathrm{M}=84.60)$, seguido de los que pertenecían a la EPS $(\mathrm{M}=75.42)$; los que menos dolor percibían fueron aquellos que contaban con medicina prepagada $(\mathrm{M}=37.67)$.

Como se puede observar, todas las variables sociodemográficas, a excepción de la variable "género", se asociaron a algunas de las variables bajo estudio (diagnóstico, ansiedad, depresión, riesgo de dependencia, control, autonomía, placer, autorrealización, calidad de vida, actividades de la vida diaria, resiliencia y dolor); sin embargo, la variable que tuvo más diferencias significativas con la mayoría de las variables del estudio fue el sistema de salud al que se encontraba adscrito el participante.

\section{Correlación entre las variables de salud}

Para el análisis de correlación entre las variables de salud (calidad de vida, resiliencia, ansiedad/depresión) se empleó el coeficiente de correlación no paramétrico de Sperman, esto debido a que ninguna de las variables tenía una distribución normal.

Se encontraron relaciones significativas positivas entre la escala de calidad de vida con resiliencia, y entre las variables de ansiedad y depresión de la escala PHQ-4. Por otra parte, hubo correlaciones significativas negativas entre ansiedad y la 
Tabla 3. Coeficiente de correlación entre calidad de vida, resiliencia, ansiedad y depresión.

\begin{tabular}{|l|l|}
\hline \multicolumn{1}{|c|}{ CAPS-19: } & \multicolumn{1}{c|}{ Resiliencia - CLHLS } \\
\hline Control & rho $=0.60: p<0.01$ \\
\hline Autonomía & rho $=0.53: p<0.01$ \\
\hline Placer & rho $=0.39: p<0.01$ \\
\hline Autorrealización & rho $=0.58: p<0.01$ \\
\hline CAPS-19: Escala total & rho $=0.70: p<0.01$ \\
\hline PHQ-4: Ansiedad & rho $=-0.35: p<0.01$ \\
\hline PHQ-4: Depresión & rho $=-0.48: p<0.01$ \\
\hline PHQ-4: & PHQ-4: Depresión \\
\hline Ansiedad & rho $=0.38 . p<0.01$ \\
\hline PHQ-4: & CAPS-19: Control \\
\hline Ansiedad & rho $=-0.32: p<0.01$ \\
\hline Depresión & rho $=-038: p<0.01$ \\
\hline PHQ-4: & CAPS-19: Autorrealización \\
\hline Depresión & rho $=-0.35: p<0.01$ \\
\hline PHQ-4: & CAPS-19: Escala Total \\
\hline Depresión & rho $=-0.40: p<0.01$ \\
\hline
\end{tabular}

dimensión de control del CAPS-19 (rho $=-.32$ : $p<0.01$ ), ansiedad y resiliencia ( $\mathrm{rho}=-.35: p<.01$ ), depresión y la dimensión de control del CAPS-19 (rho $=-.32: p<.01)$, depresión y la dimensión de autorrealización del CAPS-19 (rho $=-.35: p<.01$ ), depresión y la escala total del CAPS-19 (rho $=-.40$ : $p<.01$ ) y depresión y resiliencia (rho $=-.48$ : $p<.01)$.

Como se puede observar en las relaciones negativas, la mayoría se encontró asociada a las variables de depresión y ansiedad, mostrando, por ejemplo, que entre mayores son los puntajes de depresión, menores son los puntajes en autorrealización, resiliencia y las dimensiones del CAPS- 19 .

\section{DISCUSIÓN}

En este estudio participaron 150 adultos mayores, con edades comprendidas entre 60 y 98 años, residentes en la ciudad de Bogotá, de sexo femenino y masculino, de estratos bajos y medios-bajos en su mayoría. Respecto a las variables ansie$\mathrm{dad} /$ depresión, medidas con el PHQ-4, los niveles fueron bajos si se comparan estos resultados con las medias originales alemanas de Kroenke et al. (2009) y la versión colombiana de Kocalevent et al. (2014). Con relación a los niveles de dependencia, incapacidad y dolor, se encontraron bajos niveles en esta muestra, lo que se puede relacionar con los bajos niveles de emociones negativas halladas. Al respecto, diferentes investigaciones han encontrado que la mayor prevalencia de síntomas depresivos y ansiosos se asocia con altos niveles de dependencia por parte del adulto mayor para realizar las actividades diarias, así como con dolor (Cardona et al., 2012).

Por otro lado, con relación a la variable de resiliencia en adultos mayores, medida con el cuestionario CLHLS, las puntuaciones fueron altas y ligeramente superiores a las de las investigaciones originales en China con el cuestionario de Zeng y Shen (2010), lo que posiblemente indica que parte de la muestra de adultos mayores del presente estudio desarrollan un proceso de envejecimiento exitoso y positivo, lo que constituiría un componente importante de ajuste psicosocial, que se ha visto vinculado en la literatura científica con la salud y el bienestar de los mayores (Cárdenas y López, 2011).

Finalmente, respecto a la variable de calidad de vida medida con el CASP-19, si se comparan los presentes resultados con los del estudio de Scholes et al. (2009), desarrollado en Gran Bretaña con una muestra superior a 6,000 adultos mayores de 60 años, se aprecia que la dimensión "control" del estudio presente alcanzó puntuaciones medias ligeramente superiores $(M=7.87)$ a las de los ingleses $(M=7.61)$, lo que implica que los adultos mayores colombianos tienen una buena capacidad para participar activamente en su entorno. Respecto a la dimensión "autonomía", las puntuaciones medias obtenidas aquí fueron inferiores $(\mathrm{M}=8.96)$ a las de los ingleses $(\mathrm{M}=9.74)$, lo que significa que los adultos mayores colombianos tienen más interferencias externas para hacer cosas que desean hacer. En cuanto a la dimensión "placer", las puntuaciones medias de este estudio $(\mathrm{M}=10.04)$ fueron superiores a las de los ingleses $(M=7.61)$, lo que indica que los adultos mayores colombianos son felices y capaces de comprometerse con la vida. En lo relativo a la dimensión "autorrealización", las puntuaciones medias obtenidas ( $\mathrm{M}=$ 13.09) fueron ligeramente inferiores a las de los ingleses $(M=13.17)$, significando que los adultos mayores colombianos sienten que pueden llevar 
a cabo sus potenciales. Según la escala total del CASP-19, las puntuaciones medias de este estudio $(\mathrm{M}=39.96)$ fueron ligeramente inferiores a las de los ingleses $(\mathrm{M}=40.96)$, lo que implica que los adultos mayores colombianos expresan en términos generales una opinión favorable del proceso de envejecimiento y reconocen los aspectos positivos de la vida. De hecho, Sinisterra (2007) plantea que la población colombiana muestra altos niveles en bienestar, felicidad y optimismo, independientemente de los acontecimientos negativos o positivos que tenga que atravesar

Por otro lado, el dato de que los hombres de este estudio son más ansiosos que las mujeres va en contra con gran parte de la literatura científica en el área, ya que a pesar de que la ansiedad es común en la edad adulta, la investigación basada en la evidencia plantea que las mujeres y las personas con menos educación son más ansiosas que los hombres y los individuos con mayor nivel educativo (Arenas y Puigcerver, 2009; Ministerio de Salud y Protección Social, Colciencias, Pontificia Universidad Javeriana, y Datos, Procesos y Tecnología SAS, 2015). Al respecto, podría plantearse como una hipótesis que los niveles más bajos de ansiedad encontrados en la muestra femenina del presente estudio podrían estar asociados al hecho de que se constituyó en su mayoría por personas con una edad en la que a la mujer, en Colombia, se le asigna culturalmente un alto estatus matriarcal en las redes familiares y en grupos humanos numerosos, en los que se da y se recibe un amplio apoyo social a lo largo de la vida (Gutiérrez de Pineda, 2000). Entre los hombres colombianos, por el contrario, se enfatiza más la autonomía, la autoconfianza y la independencia, lo que los lleva a tener más dificultades al buscar la ayuda de otros ante sucesos estresantes. Sería interesante llevar a cabo en Colombia más estudios para analizar el peso diferencial que tiene el apoyo social según el sexo y su relación con emociones negativas en la tercera edad.

En cuanto a la relación entre el trabajo y la calidad de vida, no hay en la literatura demasiadas investigaciones (Amorim, Salla y Trelha, 2014). En este estudio se encontró que las personas de la tercera edad que trabajaban tenían una mejor calidad de vida que quienes no lo hacían. Estos resultados concuerdan con los de algunos estudios en el área, donde se ha hallado que la participación en actividades productivas de adultos mayores reduce las posibilidades de desarrollar fragilidad, discapacidad y depresión, y aumenta el bienestar y la satisfacción, mejorando asimismo las funciones cognitivas (Jung, Gruenewald, Seeman y Sarkisian, 2009; Schwingel, Niti, Tang y Ng, 2009).

Por otro lado, fue posible observar que las personas que vivían con amigos o familiares tenían mejor calidad de vida que los que vivían solos. A este respecto, los efectos negativos de la soledad en la salud y la calidad de vida han sido suficientemente estudiados por autores como Hawkley y Cacioppo (2010), Courtin y Knapp (2015) y Gerst-Emerson y Jayawardhana (2015).

En esa misma línea, según un estudio desarrollado en Inglaterra por Netuveli, Wiggins, Hildon, Montgomery y Blane (2005), cuyo objetivo fue investigar si se podía predecir la calidad de vida de los adultos mayores dependiendo del contexto social y las circunstancias socioeconómicas, se encontró que tener buenas relaciones con personas cercanas aumenta la calidad de vida. Vivir en un buen vecindario con amigos cercanos tiene ese mismo efecto; sin embargo, cuidar a un familiar o el hogar pueden reducir significativamente la calidad de vida.

Respecto a las relaciones entre la resiliencia y estar afiliado a los diferentes servicios de salud colombianos, se halló que los adultos mayores con menores recursos económicos, afiliados al SISBEN eran menos resilientes, en comparación con los afiliados a sistemas privados de salud (EPS y medicina prepagada), lo que lleva a inferir, por un lado, que los servicios de salud contributivos pueden proporcionar una mejor calidad en la atención, lo que aumenta la resiliencia para afrontar las adversidades de la vejez, y por otro, que estar inscrito en el SISBEN puede producir un deterioro de las capacidades de resiliencia de sus afiliados porque estos últimos se ven enfrentados a la amenaza continua de un servicio médico que podría no satisfacer las necesidades de salud, lo que llevaría a los ancianos a resistir continuamente a fin de lograr la supervivencia. Al respecto, en un estudio desarrollado con 546 adultos mayores, con una edad promedio de 75 años, en cinco centros de salud en la ciudad brasileña de Florianópolis (Hardy, Concato y Gill, 2004), se encontró que 
quienes tenían vida social, educación superior, mayor poder adquisitivo, independencia en las actividades instrumentales de la vida diaria y niveles altos de actividad física eran los que tenían los valores más altos de resiliencia. Esos mismos resultados se obtuvieron en el presente estudio entre estar afiliado al SISBEN, una percepción más negativa de calidad de vida y más ocurrencia de dolor, al igual que un cierto grado de relación con una investigación desarrollada en Colombia por el Departamento Nacional de Planeación (DPN) (2016), en el que se halló que las personas afiliadas al régimen contributivo de salud son más felices y menos deprimidas que las personas afiliadas al régimen subsidiado, así como con el estudio de Torres, Méndez, López, Galarza y Oviedo (2013), quienes hallaron que las condiciones sanitarias deficientes en la ciudad de Bogotá eran una variable suficiente para clasificar la calidad de vida de la población.

Finalmente, la correlación positiva encontrada entre la resiliencia con todas las dimensiones de calidad de vida medidas con el CAPS-19 confirma el valor protector de esta variable salutogénica encontrada en numerosos estudios con adultos mayores (Fernandes de Araujo, Teva y Bermúdez, 2015; MacLeod, Musich, Hawkins, Alsgaard y Wicker, 2016); por el contrario, la correlación negativa observada entre las variables de ansiedad y depresión con todas las dimensiones de calidad de vida confirma el valor patogénico de estas dos emociones negativas en tales sujetos (Gerini, Marino, Brustia, Lyrakos y Rolle, 2015).

Entre las limitaciones del estudio se halla no haber incluido en él personas institucionalizadas, así como el tamaño de la muestra reducido de 150 personas residentes en la ciudad de Bogotá, lo cual se suma el que la literatura científica en el área sea ciertamente escasa, por lo que se recomienda llevar a cabo más estudios sobre el referido problema en el contexto nacional y latinoamericano. Los resultados de este estudio llevan a sugerir la puesta en práctica de programas que potencien emociones positivas, relajación, meditación y entrenamiento en técnicas de autocontrol en adultos mayores (cf. Quiceno, Remor y Vinaccia, 2016; Petretto, Pili, Gaviano, Matos y Zuddas, 2016), con el propósito de desarrollar procesos para un envejecimiento exitoso.

\section{REFERENCIAS}

Agudelo, L., Ayala, M.L. y Ríos, M.L. (2009). Caracterización de la movilidad de ancianos según el índice de Katz en el centro de bienestar del anciano San José de Pereira. Investigaciones Andina, 11, 69-79.

Alexandre N., M.C. y Guirardello E., B. (2002). Adaptación cultural de instrumentos utilizados en salud ocupacional. Revista Panamericana de Salud Pública, 11(2), 109-111.

Álvarez, M., De Alaiz A., T., Brun, E., Cabañeros, V., Calzón, M., Cosío, I. et al. (1992). Capacidad funcional de pacientes mayores de 65 años, según el Índice de Katz. Fiabilidad del método. Atención Primaria, 10(6), 812-815.

Amorim, J.S.C., Salla, S. y Trelha, C.S. (2014). Factors associated with work ability in the elderly: systematic review. Revista Brasileira de Epidemiologia, 17, 830-841.

Arenas, M.C. y Puigcerver, A. (2009). Diferencias entre hombres y mujeres en los trastornos de ansiedad: Una aproximación psicobiológica. Escritos de Psicología, 3(1), 20-29.

Bacca A., M., González, A. y Uribe A., F. (2005). Validación de la Escala de Depresión de Yesavage (versión reducida) en adultos mayores colombianos. Pensamiento Psicológico, 1(4), 53-63.

Barber, J.H., Wallis, J.B. y McKeating, E. (1980). A postal screening questionnaire in preventive geriatric care. The Journal of the Royal College of General Practioners, 30(210), 49-51.

Bennett, K., Reyes, F., Altamar, P. y Soulsby, L. (2016). Resilience amongst older Colombians living in poverty: an ecological approach. Cross Cultural Gerontology, 31(4), 385-407.

Cano C., A., Borda M., G., Arciniegas A., J. y Parra J., S. (2014). Hearing disorders in elderly people, associated factors and quality of life: SABE study. Biomedica, 34(4), 574-579.

Cárdenas J., A. y López D., A.L. (2011). Resiliencia en la vejez. Revista de Salud Pública, 13(3), 528-540.

Cardona, D., Estrada, A. y Agudelo, H. (2006). Calidad de vida y condiciones de salud de la población adulta de la ciudad de Medellín. Biomédica, 26, 206-215. 
Cardona J., A., Álvarez M., I. y Pastrana, S. (2012). Calidad de vida relacionada con la salud en adultos mayores de hogares geriátricos de Medellín. Revista de Ciencias de la Salud, 12(2), 139-155.

Cornwell, E.I. (2016). Household disorders, network ties, and social support in later life. Journal Marriage Family, 78(4), 871-889.

Courtin, E. y Knapp, M. (2015). Social isolation, loneliness and health in old age: a scoping review. Health Social Care Community, 25(3), 799-812. DOI: 10.1111/hsc.12311.

Curcio, C.L., Henao, G.M. y Gómez, F. (2014). Frailty among rural elderly adults. BMC Geriatry, 10, 14-20.

Departamento Nacional de Planeación (2016). ¿Qué es la felicidad? Primer diagnóstico para Colombia. Bogotá: DPN.

Dulcey, E., Arrubla, D. y Sanabria, P. (2013). Envejecimiento y vejez en Colombia. Bogotá: Profamilia

Fedeli, D. (2014). L'invecchiamento psicológico. Il mantenimento della qualita della vita (QvD) e il modelo "Proactive Aging". Oikonomia, 11(3), 11-14.

Fernandes de Araujo, L., Teva, I. y Bermúdez, M.P. (2015). Resilience in adults: a teorical review. Terapia Psicológica, 33(3), 257-266.

Fontes, A. y Neri, A. (2015). Resilencia y velhice: revisao de literatura. Ciencia \& Saúde Coletiva, 20(5), 1475-1495.

Fundación Saldarriaga Concha (2015). Misión Colombia envejece. Bogotá: FSC.

Gerini, E., Marino, E., Brustia, P., Lyrakos, P. y Rolle, L. (2015). Quality of life in the third age: a research on risk and the protective factors. Procedia. Social and Behavioral Sciences, 187, 217-222.

Gerst-Emerson, K. y Jayawardhana, J. (2015). Loneliness as a public health issue: the impact of loneliness on health care utilization among older adults. American Journal o Public Health, 105(5), 1013-1019. DOI: 10.2105/AJPH.2014.302427.

Global Aging Institute (2017). Global aging and outlook for grow and stability in the developing word. Alexandria, VA: Author.

Gómez, F., Corchuelo, J., Curcio, C.L., Calzada, M.T. y Méndez, M.T. (2016). SABE Colombia: Survey on health, well-being and aging in Colombia. Study design and protocol. Current Gerontology and Geriatrics Research, 24, 1-7.

Gómez, F., Curcio, C.L. y Duque, G. (2009). Health care for older's persons in Colombia: A country profile. Journal of the American Geriatrics Society, 57(9), 1692-1696.

Gómez, J., Curcio, L. y Henao, G.M. (2012). Fragilidad en ancianos colombianos. Revista Médica Sanitas, 15, 8-16.

Gutiérrez de Pineda, V. (2000). Modalidades familiares de fin de siglo. En V. Gutiérrez de Pineda, J. Aldana, G. Pérez y M. I. Velasco (Eds.): La familia en la perspectiva del año 2000 (pp. 9-27). Bogotá: Magisterio.

Hardy, S.E., Concato, J. y Gill, T.M. (2004). Resilience of community-dwelling older persons. Journal of the American Geriatrics Society, 52(2), 257-262.

Hawkley, L. y Cacioppo, J. (2010). Loneliness matters: a theorical and empirical review of consequences and mechanisms. Annals of Behavioral Medicine, 40(2), 1-6.

Hernández, R., Fernández, C. y Baptista, P. (2010). Metodología de la investigación. México: McGraw-Hill.

Hoogendijk, D., Deeg, D., Poppelars, J., Van de Horst, M., Broese, M., Comisjs, H. y Huisman, M. (2016). The longitudinal aging study Amsterdam: cohort update 2016 and mayor findings. Aging Clinical Experimental Research, 31(9), 927-945.

Hyde, M., Wiggins, R.D., Higgs, P. y Blane, D.B. (2003). A measure of quality of life in early old age: the theory, development and properties of a needs satisfaction model (CASP-19). Aging and Mental Health, 7, 86-94.

Jung, Y., Gruenewald, T.L., Seeman, T.E. y Sarkisian, C.A. (2009). Productive activities and development of frailty in older adults. Journal of Gerontology, 65(2), 256-261.

Kahana, E., Kelley-Moore, J. y Kahana, B. (2012). Proactive aging: A longitudinal study of stress, resources, agency, and well-being in late life. Aging \& Mental Health, 16(4), 438-451.

Katz, S., Ford, A.B., Moskowitz, R.W., Jackson, B.A. y Jaffe, M.W. (1963). Studies of illness in the age: The index of ADL, a standarized measure of biological and psychosocial function. Journal of the American Medical Association, 185, 914-919.

Kocalevent, R.D., Finck, C., Jimenez-Leal, W., Sautier, L. y Hinz, A. (2014). Standardization of the Colombian version of the PHQ-4 in the general population. BMC Psychiatry, 14(205). DOI: 10.1186/1471-244X-14-205.

Kroenke, K., Spitzer, R.L., Williams, J.B. y Lowe, B. (2009). An ultra-brief screening scale for anxiety and depression: The PHQ-4. Psychosomatics, 50, 613-621.

Larragoita, A. y Larragoita, I. (1996). Adaptación en nuestro medio de una encuesta para la detección de ancianos con riesgo de dependencia. Gerontología, 6, 224-231.

Lucumi, D., Gomez, L., Browson, R. y Parra, D. (2014). Socioeconomic and health-related quality of life among older adults in Bogotá (Colombia). Journal of Aging and Health, 27(4), 730-750.

MacLeod, S., Musich, S., Hawkins, K., Alsgaard, K. y Wicker, E. (2016). The impact of resilience among older adults. Geriatric Nursing, 37, 266-272.

Ministerio de Salud y Protección Social, Colciencias, Pontificia Universidad Javeriana y Datos, Procesos y Tecnología SAS (2015). Encuesta Nacional de Salud Mental 2015. Bogotá: Autores. 
Netuveli, G., Wiggins, R.D., Hildon, Z., Montgomery, S.M. y Blane, D. (2005). Functional limitation in long standing and quality of life: evidence from a national survey. British Medical Journal, 331(7529), 1382-1383.

Nimrod, G. y Shrira, A. (2016). The paradox of leisure in later life. Journal of Gerontology, 71(1), 106-111.

Ocampo, J.M., Zapata, J., Cubides, A.M., Curcio, C. y Villegas, I. (2013). Prevalence of por self-rated healthand associated risk factors among older adults in Cali, Colombia. Colombia Médica, 44(4), 224-231.

Organización Mundial de la Salud (2015). Informe Mundial de Envejecimiento y Salud. Ginebra: OMS.

Petretto, D., Pili, R., Gaviano, L., Matos, C. y Zuddas, C. (2016). Envejecimiento activo y de éxito o saludable: una breve historia de modelos conceptuales. Revista Española de Geriatría y Gerontología, 51(4), 229-241.

Poli, S., Cella, A., Puntoni, M., Musacchio, C., Pomata, M., Torriglia, D. y Pilotto, A. (2016). Frailty is associated whith socioeconomic and lifestile factors in community-dewlling older subjecs. Aging Clinical and Experimental Research, 29(4), 721-728. doi: 10.1007/s40520-016-0623-5.

Quiceno, J.M., Remor, E. y Vinaccia, S. (2016). FORTALEZA: Programa de Potenciación de la Resiliencia para Promoción y el Mantenimiento de la Salud. México: El Manual Moderno.

Scholes, S., Medina, J., Cheshire, H., Cox, K., Hacker, E. y Lessof, C. (2009). Technical report (wave 3): The 2006 English Longitudinal Study of Ageing. London: London National Centre for Social Research.

Schwingel, A., Niti, M.M., Tang, C. y Ng, T.P. (2009). Continued work employment, volunteerism, and mental well-being of older adults: Singapore longitudinal ageing studies. Age \& Ageing, 38(5), 531-537.

Sinisterra, D. (2007). Colombian happiness: A look at life satisfaction and explanatory style. Disertación Doctoral no publicada. Boca Raton, FL: Florida Atlantic University.

Smith, J.L. y Hollinger-Smith, L. (2015). Savoring, resilience, and psychological well-being in older adults. Aging \& Mental Health, 19(3), 192-200.

Torres, A., Méndez, S., López, L., Galarza, S. y Oviedo, N. (2013). Calidad de vida y ciudad: análisis del nivel de desarrollo en Bogotá a través del método de las necesidades básicas insatisfechas. Estudios Generales, 29, 231-238.

Vinaccia, S. (2010). Psicología de la salud: análisis exploratorio de instrumentos. Manuscrito no publicado. Madrid: Universidad Autónoma de Madrid.

Zeng, Y. y Shen, K. (2010). Resilience significantly contributes to exceptional longevity. Current Gerontology and Geriatrics Research, 10, 1-9. DOI: 10.1155/2010/525693. 